\title{
Rivaroxaban and its Use in the Elderly, What Do We Know?
}

\section{Kenneth T. Moore*}

Department of Cardiovascular Disease and Metabolism, Janssen Pharmaceuticals, USA

\section{Opinion}

With an ever-increasing elderly population, comes the demand for newer treatment options that can be safely used. However, older individuals are poorly represented in clinical trials during drug development. Thankfully, many of the pharmaceutical companies are becoming more sensitive to the need for obtaining greater information in these under represented populations. For example, clinical trials like ROCKET-AF (Rivaroxaban Once Daily Oral Direct Factor X Inhibition Compared with Vitamin K Antagonism for Prevention of Stroke and Embolism) included 14,264 patients with non-valvular atrial fibrillation (NVAF) at moderate-to-high risk of stroke, of which $44 \%$ were comprised of patients $\geq 75$ years of age $[1,2]$.

This is relevant considering that the use of non-vitamin K antagonist oral anticoagulants (NOACs) like rivaroxaban, has quickly established a strong footing in the management of thromboembolic disease and provides another easier option to a treatment armory that has been historically dominated by vitamin $\mathrm{K}$ antagonists like warfarin. While the use of warfarin has been successful across different disease states and patient populations, it does have some intrinsic pharmacological properties that make it difficult to manage, particularly for older individuals.

However, is data obtained from randomized clinical trials enough? Yes, for approval by regulatory bodies like the United States Food and Drug Administration or the European Medicines Agency, where the wealth of data collected from pre-clinical animal studies through large pivotal patient clinical trials establishes the basis of our understanding. However, many companies don't rest there. They continue to study the drug long after its initial approval, collecting data for new indications, different populations or confirming the safety and efficacy of the original clinical trials conducted.

It is important to note that the pharmaceutical industry is not alone in this effort. Independent researchers from academia also contribute to the wealth of data reported. Analysis of 'Real World Data', which is typically insurance claims data collected after the drug has been approved and marketed, is one such source of information.

In the case of rivaroxaban and its use in an older population, work done by Martinez et al. [3] and Coleman et al. [4] reported on the real- world efficacy and safety of rivaroxaban in 'Frail' patients with NVAF and VTE, respectively. The work done by Martinez showed that in this population, rivaroxaban was associated with a non-significant reduction in the hazard of developing a stroke, systemic embolism or ischemic stroke at 1-year follow-up when compared to warfarin and had similar rates of bleeding along with a significant reduction in intracranial hemorrhage [3]. Remarkably, at the 2-year follow-up, rivaroxaban was associated with a significant reduction in the hazard of developing a stroke, systemic embolism or ischemic stroke compared to warfarin, with no significant difference in bleeding [3].

The results from Coleman et al. regarding the treatment of frail patients with VTE shows a consistent message to that of Martinez. The results of the Coleman study show that rivaroxaban reduced the hazard of the composite of recurrent VTE alone compared to warfarin. Additionally, there was no significant difference in major bleeding [4].

In the absence of a clinical trial conducted entirely in elderly or frail patients and notwithstanding the unique challenge of comorbidities, changing physiology, concomitant medications and dietary and physical limitations that come with advancing age, the body of pharmacological, clinical trial data and real-world evidence collected to date, supports the safe and effective use of rivaroxaban in older patients when compared to warfarin. That being said, a cautious and individualized approach (assessing the risk/benefit of treatment) is always prudent in this population. Lastly, future trials for rivaroxaban and any other therapy ultimately utilized in an elderly population, needs to empirically evaluate these types of populations.

\section{References}

1. Patel MR, Mahaffery KW, Garg J (2011) Rivaroxaban versus warfarin in nonvalvular atrial fibrillation. N Engl J Med 365: 883-891.

2. Moore KT, Wong $P$, Zhang $L$ (2018) Influence of age on the pharmacokinetics, pharmacodynamics, efficacy, and safety of rivaroxaban. Curr Med Res Opin Aug 5: 1-9.

3. Martinez B, Sood N, Bunz T (2018) Effectiveness and safety of apixaban dabigatran, and rivaroxaban versus warfarin in frail patients with nonvalvular atrial fibrillation. J Am Heart Assoc 7: e008643.

4. Coleman Cl, Turpie AGG, Bunz TJ (2018) Effectiveness and Safety of rivaroxaban versus warfarin in frail patients with thromboembolism. Am J Med 13: 933-938.
${ }^{*}$ Corresponding author: Kenneth T. Moore, Scientific Director of Medical Affairs, Department of Cardiovascular Disease and Metabolism, Janssen Pharmaceuticals Inc. 1125 Trenton-Harbourton Road, Titusville, NJ 08560, USA, Fax: 609730-6452; Tel: 732822-9754; E-mail: tmoore17@its.jnj.com

Received August 23, 2018; Accepted August 28, 2018; Published August 31, 2018

Citation: Moore KT (2018) Rivaroxaban and its Use in the Elderly, What Do We Know? J Gerontol Geriatr Res 7: 478. doi:10.4172/2167-7182.1000478

Copyright: (c) 2018 Moore KT. This is an open-access article distributed under the terms of the Creative Commons Attribution License, which permits unrestricted use, distribution, and reproduction in any medium, provided the original author and source are credited. 